\title{
DESARROLLO SOSTENIBLE Y EL IMPACTO EN EL MEDIO AMBIENTE
}

\section{SUSTAINABLE DEVELOPMENT AND THE IMPACT ON THE ENVIRONMENT}

\author{
DOI: Https://doi.org/10.53595/rlo.2021.1.007
}

\author{
Jaime Quispe Charca \\ Universidad Nacional del Altiplano \\ https://orcid.org/0000-0003-2913-5657 \\ jeanelias1289@gmail.com
}

\section{RESUMEN}

En el presente ensayo se revisa la problemática que en el transcurso de los años ha generado el desarrollo sostenible, por tal motivo fue objeto de controversia, cuestionamientos y diversas posturas frente a la evolución de la misma. En muchos casos, las malas prácticas en los procesos generan un deterioro de recursos vitales como el agua, los bosques y la tierra, los cuales se renuevan a un ritmo más lento del que son explotados. Todo ello tiene un impacto negativo en el que las medidas deben ser tomadas para afrontarlo de la mejor manera posible. El desarrollo sostenible se refiere a un prototipo de desarrollo que utilice los recursos disponibles en el presente, sin comprometer su existencia en el futuro. Por tanto se requiere de un desarrollo sostenible que satisfaga las necesidades del presente sin poner en peligro a las generaciones futuras para atender sus propias necesidades, por ello la preocupación por la pérdida de la biodiversidad y el agotamiento de los recursos renovables, la contaminación del agua, ríos, el aire, el deterioro de la capa de ozono, la erosión y la deforestación. Donde la informalidad o los vacíos legales o falta de los mismos, así como los inexistentes sistemas de gestión ambiental, traen como consecuencia terribles impactos ambientales. A partir de allí surge la necesidad de relacionar algunos elementos clave, así como los diferentes puntos del desarrollo sostenible y medio ambiente que es vida.

Palabras clave: Desarrollo sostenible y medio ambiente.

\section{ABSTRACT}

This essay reviews the problem that sustainable development has generated over the years, for this reason it was the subject of controversy, questions and various positions regarding its evolution. In many cases, bad practices in the processes generate a deterioration of vital resources such as water, forests and land, which are renewed at a slower rate than they are exploited. All this has a negative impact in which measures must be taken to deal with it in the best possible way. Sustainable development refers to a prototype of development that uses the resources available in the present, without compromising their existence in the future. 
Therefore, a sustainable development is required that meets the needs of the present without endangering future generations to meet their own needs, hence the concern for the loss of biodiversity and the depletion of renewable resources, water pollution , rivers, the air, the deterioration of the ozone layer, erosion and deforestation. Where informality or legal loopholes or lack thereof, as well as non-existent environmental management systems, result in terrible environmental impacts. From there, the need arises to relate some key elements, as well as the different points of sustainable development and the environment that is life.

Keywords: Sustainable development and environment.

\section{INTRODUCCIÓN}

Los problemas ambientales no son nuevos, el hombre desde siempre ha interactuado con el medio y lo ha modificado con sus actividades. En la actualidad existen debates que asumen el desarrollo como una suma de aspectos cuantitativos y cualitativos, sin embargo en los centros de decisión se sigue defendiendo el crecimiento económico como la vía para conseguir el desarrollo. La noción de crecimiento se ha manifestado como muy importantes, se asimilo producción y consumo con desarrollo ignorando otras magnitudes que con el tiempo se han manifestado como muy importantes, el paradigma económico imperante equipara crecimiento económico con desarrollo y pleno empleo lo que obliga a un crecimiento económico constante y esto va a ser la marca de un éxito tanto de países, empresas y personas, en la década de los 60 los problemas ecológicos empiezan a ser patentes y son múltiples las voces que nos hablan de ello y se recogen en distintos documentos.

Los problemas ambientales no son nuevos, el hombre desde siempre ha interactuado con el medio y lo ha modificado con sus actividades. En la décadas de los 80 son problemas visibles y se observan problemáticas globales como la deforestación de la desertificación y contaminación ambiental en sus diversos modos y de otros problemas que ya se empiezan a manifestarse como muy relevantes como el calentamiento global y cambio climático.

La actividad humana está dañando seriamente los sistemas que sostienen y mantienen la vida y la idea de crecimiento que está basado nuestra e economía en uso creciente de materias primas y energía, se basa en el crecimiento como un fin en sí mismo, y no tanto en alcanzar el bienestar que realmente es la idea de desarrollo sostenible. Comienzan de esta manera a parecer un cuestionamiento del modelo económico en el que la economía es el centro y los sistemas ecológicos de nuestro medio ambiente, están fuera de este sistema económico que los utiliza en su propio provecho. 
En esta década la economía es parte de un sistema que esta insertado dentro de las sociedades, y nuestro medio ambiente que le sirve de base y sustento, asimismo los modelos de desarrollo actual favorecen la pobreza y desigualdad, como también existe una relación entre los modelos de desarrollo y la situación actual de crisis ambiental, crisis social, y el concepto de necesidades, todo esto básico a la hora de hablar de sostenibilidad, de otra parte los límites en nuestras formas de consumir, formas de vida, por lo tanto un cuestionamiento de los sistemas económicos y sistemas predominantes en las sociedades y las necesidades de un cambio de paradigma hacia un sistema que realmente replantee nuestras bases éticas de vida consumo y cuidado de nuestro medio ambiente.

\section{DESARROLLO}

\section{Desarrollo humano}

El cambio más significado en el concepto de desarrollo se debe a las propuestas del desarrollo humano. En el marco del sistema de las Naciones Unidas, el Programa de Naciones Unidas para el desarrollo (PNUD) lanzó a finales de los ochenta el paradigma del desarrollo humano. La formulación de paradigmas del desarrollo humano se manifiesta en el ambiente, que se ha caracterizado por plantear el crecimiento económico como objetivo. Por el contrario, el desarrollo humano aclara que éste no debe ser el objeto central del desarrollo sino uno de sus referentes. Su propuesta supone un cambio radical de los planteamientos anteriores en dos sentidos: Uno, porque coloca el centro de la concepción del desarrollo en el proceso de expansión de las capacidades de las personas de manera que puedan elegir su modo de vida. Dos, porque cuestiona que el desarrollo dependa fundamentalmente de la expansión del capital físico y resalta lo importante del capital humano. En resumen, se ve una visión del desarrollo y elaboración en la producción de bienes por otra parte en la ampliación de las capacidades de las personas. Aunque no se puede afirmar que el PNUD haya sido su descubridor del desarrollo humano, no deja de ser menos importantes sus aciertos, conceptos y sus informes que han servido como base para nuevos conceptos, nuevas ideas y han conseguido convertirlo en un referente.

En la actualidad se habla mucho con estas bases sobre el desarrollo humano. Pero el paradigma del desarrollo humano no se limita a las propuestas específicas que el PNUD realiza, por muy significativas e importantes que sean éstas, otras agencias de desarrollo internacionales y nacionales, así como muchas organizaciones no gubernamentales y en el pensamiento académico, se trabaja en la elaboración de importantes propuestas 
estimuladas en los fundamentos del desarrollo humano. En la elaboración de este enfoque de desarrollo, hay que mencionar la figura del premio Nobel de Economía, 1998, Sen, cuyas críticas al concepto de bienestar basado en la acumulación, o en la opulencia, como expresa muy certeramente, y su propuesta de un bienestar centrado en la persona humana, han tenido un amplio eco.

Entonces el enfoque de desarrollo humano impulsado por el PNUD, también se inspira, y así lo reconoce como tal, en sus aportaciones teóricas. Hace mucho tiempo la pregunta importante en torno al desarrollo era: ¿Cuánto produce una nación?; ahora la pregunta central pasa a ser: ¿cómo está la gente? Las formulaciones del desarrollo humano se han encontrado, su expresión más conocida en los Informes del PNUD. Recogemos la siguiente cita del Informe correspondiente al año 2000, como ejemplo de la nueva formulación del desarrollo: "El desarrollo humano es el proceso de ampliación de las opciones de la gente, aumentando las funciones y las capacidades humanas... Representa un proceso a la vez que un fin. Entonces en todos los niveles de desarrollo de las tres capacidades principales permiten que las personas vivan una larga vida y saludable, que tengan diez conocimientos y accesos a recursos básicos para que tengan un nivel de vida decente.

Pero el ámbito del desarrollo humano va más allá: existen otras alternativas que las personas consideran en alta medida la participación, la seguridad, la sostenibilidad, las garantías de los derechos humanos, todos estos puntos son muy importantes y necesarias para ser creativo y productivo y para gozar de respeto mutuo, y tener una sensación de acercamiento a una comunidad. Entonces, el desarrollo humano es el desarrollo de las personas, para la gente y por la gente." El desarrollo, desde este punto de vista, ha dejado de ser una simple técnica para conseguir algunos resultados. Es algo mucho más grande, que por supuesto también necesita de esas técnicas.

El desarrollo recupera toda la magnitud del futuro y de la creatividad humana: Está llena de tensiones, en la que hay que determinar qué bienes y servicios se necesitan o se desean, porque ello son los más importantes, que materiales serán necesarios y los más indicados, etc. Y responder a estas preguntas exige tener una concepción ética de cuál es la vida que se quiere formar, cuáles son las relaciones entre las personas que se consideran las más apreciadas e importantes, en definitiva, se basa en tener mayor preocupación por el desarrollo y por misma situación que contenga justicia, porque si no funciona así, difícilmente se le podrá considerar humano. 
El enfoque del desarrollo humano cuestiona el que exista una relación directa entre el crecimiento del ingreso y la ampliación de las acciones que se ofrecen a las personas. No es suficiente con analizar la cantidad, sino es más importante tener en cuenta la calidad de ese crecimiento. Por eso, no es que muestre desinterés por el crecimiento económico, sino se debe resaltar la necesidad de que este crecimiento debe verificarse en función de que consiga o no que los seres humanos puedan realizarse cada vez mejor. Entonces, le preocupa que se establezcan relaciones positivas entre el crecimiento económico y las opciones de las personas.

\section{Desarrollo sostenible}

El desarrollo sostenible es una transformación de cambio creciente en la condición de vida de las personas, que se coloca como el núcleo y ente primordial del desarrollo, por medio del crecimiento económico para la igualdad social, la transformación de los procedimientos de producción y formas de consumo para poder mantener el equilibrio ecológico y el soporte vital de nuestro ecosistema.

Esta transformación implica el respeto a la biodiversidad, étnica cultural, nacional y local, así como el afianzamiento y la global participación ciudadana en la convivencia armónica con la naturaleza, sin arriesgar y asegurando la calidad de vida de las futuras generaciones. Galo Muñoz Arce, (2004).

El desarrollo sostenible implica un equilibrio entre medioambiente, productividad y la sociedad, además indica que solo las empresas que generan beneficios, creando riqueza y empleo sin poner en riesgo las necesidades sociales y ambientales de la sociedad, serán los que contribuyen con el desarrollo sostenible. Fernández (2010, p. 24).

Después de la Conferencia Internacional sobre Medio Ambiente y Desarrollo realizada el año 1972 en Estocolmo, Suecia, se dio inicio a un nuevo enfoque a una nueva visión de desarrollo sostenible; es decir, hasta ese momento solo se consideraba dos dimensiones: económico y social; pero a medida que va pasando el tiempo, los años es necesario el cuidado y la protección del medio ambiente, debido a la degradación y sobre explotación de los recursos naturales por parte del ser humano, surge la dimensión ambiental. Rojas y Parra. (2003, p. 246) y Calderón, Chumpitaz, Sumarán y Campos (2011, p. 17).

\section{Educación para el desarrollo sostenible}

El crecimiento y avance tecnológico que pasa a nivel mundial ha provocado graves consecuencias, como el incremento de las desigualdades sociales y el profundo deterioro de nuestro medio ambiente. Ante esta situación tan crítica para nosotros, estamos tomando conciencia a la necesidad de modificar y enmendar los modelos tradicionales 
de progreso y desarrollo procurando interrelaciones sostenibles entre sociedad y naturaleza. Estamos de acuerdo de que no podemos aceptar de ninguna manera una visión de crecimiento económico que genere el sistemática destrucción y degradación de los ecosistemas que sustentan la vida, amenazas a la diversidad biológica y cultural, concentración de poder y recursos en pocas manos, exclusión social, económica y política y el deterioro de la calidad de vida de grandes mayorías de la población.

Como educadores, nos corresponde en ese sentido, una responsabilidad ineludible, pues bien sabemos que la educación es clave para renovar, afianzar los valores, crear conciencia y tener mejor comprensión de los problemas que nos afectan y promover, fomentar actitudes de compromiso que posibiliten el cambio. Martínez-Carrera S, Carnicero A, Carrera I. (2021).

\section{Educación Ambiental}

"La educación ambiental pretende lograr que la población mundial tenga conciencia del medio ambiente y se preocupe por él y sus problemas además cuente con los conocimientos, aptitudes, actitudes y motivaciones y deseos necesarios para trabajar individual y colectivamente en la búsqueda de soluciones a los problemas actuales y para prevenir la aparición de otros nuevos" Galván (2009, p. 66).

A través de la educación ambiental se quiere contribuir e influenciar en la población para lograr una cantidad de conocimientos verdaderos capaces de tomar conciencia, para luego trabajar de forma individual y colectiva en la búsqueda del bien común y solucionar los problemas actuales y prevenir futuros problemas o conflictos ambientales. Basterra y Peralta (2014, p. 20).

"La educación ambiental se convierte en un proceso educativo integral, que se da en toda la vida del individuo, y busca generar en éste los conocimientos, las actitudes, los valores y las prácticas necesarios para desarrollar sus actividades ambientales adecuadas, con miras a contribuir al desarrollo sostenible del país. La Ley General del Ambiente (2005).

\section{Medio Ambiente}

El medio ambiente es el espacio en el que se desarrolla la vida, tanto de los seres bióticos como abióticos en sus diversos reinos y que permite su interacción.

\section{La contaminación ambiental}

La contaminación ambiental es todo cambio indeseable en las características del aire, el agua, el suelo o los alimentos, que afecta nocivamente a la salud, la sobrevivencia o las actividades de las personas u otros organismos vivos. La mayoría de los contaminantes son sustancias químicas sólidas, líquidas o gaseosas producidas como desechos, cuando 
un recurso primo es extraído, procesado, transformado en productos y utilizado. Miguel, Pena, Tamayo 2013, Pág. 10)

\section{Un desarrollo económico y social respetuoso con el medio ambiente}

El objetivo del desarrollo sostenible es crear proyectos viables y reconciliar los aspectos económico, social, y ambiental de las actividades humanas; se trata de progresar en estos ámbitos sin tener que destruir el medio ambiente. Los tres aspectos que deben ser tenidos en cuenta tanto por las empresas, como por las comunidades y las personas:

- Sostenibilidad económica: se da cuando la producción y la actividad se dirija hacia la sostenibilidad ambiental, social y es financieramente viable y rentable.

- Sostenibilidad social: basada en la relación social y de su habilidad para trabajar en la persecución de objetivos comunes. Implica la mitigación de impactos sociales negativos causados por la actividad que se desarrolla, así como la potencialización de los impactos positivos. Se involucra también con el hecho de que las comunidades locales, reciban beneficios por el desarrollo de la actividad realizada en aras de mejorar sus condiciones de vida.

- Sostenibilidad ambiental: compatibilidad entre la actividad considerada y la preservación de la biodiversidad y de los ecosistemas, evitando la degradación de nuestros recursos. Evitando el consumo excesivo de recursos difícil o lentamente renovables para que la sostenibilidad sea estable.

\section{La justificación del desarrollo sostenible.}

Se puede justificar con el hecho de que el ser humano habita en un planeta con recursos naturales agotables y limitados. Si la biodiversidad desaparece nosotros juntamente con este, ya que no existiría nutrientes en el suelo, agua potable, minerales, etc. y todo ello sería irreversible para nuestro medio ambiente.

\section{Condiciones para el desarrollo sostenible}

Los límites de los recursos naturales sugieren tres reglas básicas en relación con los ritmos para dicho desarrollo:

1. Ningún recurso renovable deberá utilizarse a un ritmo superior al de su generación.

2. Ningún contaminante deberá producirse a un ritmo superior al que pueda ser reciclado, neutralizado o absorbido por el medio ambiente.

3. Ningún recurso no renovable deberá aprovecharse a mayor velocidad de la necesaria para sustituirlo por un recurso renovable utilizado de manera sostenible. 
Según algunos autores, estas tres reglas están forzosamente supeditadas a la inexistencia de un crecimiento demográfico.Se llama desarrollo sostenible a aquel desarrollo que es capaz de satisfacer las necesidades actuales sin comprometer, afectar nocivamente los recursos y posibilidades de las futuras generaciones. Intuitivamente una actividad sostenible es aquella que se puede mantener. Por ejemplo, cortar árboles de un bosque asegurando la repoblación es una actividad sostenible. En cambio, consumir petróleo no es sostenible con los conocimientos actuales, ya que no se conoce ningún sistema para crear petróleo a partir de la biomasa. Hoy sabemos que una buena parte de las actividades humanas no son sostenibles a medio y largo plazo tal y como hoy en día están planteadas. A lo largo de la historia, el ser humano ha utilizado sus conocimientos del medio natural y las herramientas disponibles para extraer y transformar los elementos de la naturaleza que sirven para satisfacer sus necesidades.

\section{CONCLUSIÓN}

Contribuir a tener amplia visibilidad y ser críticos acerca del desarrollo sostenible para que puedan generar aspectos positivos y menos dañinos en la comunidad y en el medio ambiente.

Crear una conciencia ciudadana para que las futuras generaciones puedan disfrutar de un medio ambiente no contaminado. Amoldar a la población y comunidad entera para lograr una sostenibilidad adecuada conforme a planes de estudio.

La comunidad debe ser involucrada no sólo en la identificación de los problemas ambientales, sino también en las acciones orientadas a la solución de los problemas identificados; tomando en cuenta la valoración y respeto a la cultura de los pobladores, como sector educación jugamos un papel muy importante para preservar el medio ambiente para las generaciones futuras.

\section{REFERENCIAS}

Asunción, M. y Segovia, E. (2005). Educación ambiental no formal. http://www.unescoetxea.org/ext/manual/html/eanoformal.html

Bedoy, V. (2000) La historia de la Educación ambiental: reflexiones pedagógicas. http://educacion.jalisco.gob.mx/consulta/educar/13/13Bedoy.html

Calvo, S. (2006). Educación para el desarrollo sostenible: evaluación de retos y oportunidades del decenio 2005-2014. Revista Iberoamericana de Educación-40. www.oei.es/oeivirt/educambien.htm. Consultado 12 noviembre 2007.

Castillo, S. (2002) Pedagogía Ambiental. La tarea de los educadores. http://www.capaz.cl/biblioteca/mosaicos/m38/portada.htm

Flórez Yepes, G. Y. (2015). La educación ambiental y el desarrollo sostenible en el contexto colombiano. Revista Electrónica Educare, 1-12. http://www.redalyc.org/articulo.oa?id=194140994022.

Muñoz Arce, Galo. (2004), El Desarrollo Humano Sostenible

Articulo contexto latinoamericano 1-7

https://xdocs.cz/doc/el-desarrollo-humano-sostenibledocx-vo9m24plap8j 
Garrido, J. A. (2015). Consumismo y medio ambiente. EL UNIVERSAL, pág. 01. http://www.eluniversalqueretaro.mx/content/consumismo-y-medio-ambiente

Morales, I. G. (28 de Marzo de 2018). Desarrollo sostenible, un aliado del medio ambiente. EL ESPECTADOR, pág. 01. https://www.elespectador.com/noticias/medioambiente/desarrollosostenible-un-aliado-del-medio-ambiente-articulo-746782

Ramírez Ospina, D. E. (2017). DESARROLLO SOSTENIBLE COMO UN PROYECTO DE MODERNIDAD. Revista Ciencias Estratégicas $22 \quad$ (31), 67-81. http://www.redalyc.org/articulo.oa?id=151332653005.

Sen, Amartya .(2000). La teoría del desarrollo humano. Versión impresa ISSN 0188-4557

Estud. soc vol.19 no.37 México ene./jun. 2011

http://www.scielo.org.mx/scielo.php?script=sci_arttext\&pid=S018845572011000100010\#: :text=En\%20el\%20informe\%20se\%20define,para\%20satisfacer\%20sus $\%$ 20propias\%20necesidades.

UNESCO. (2014). EDUCACIÓN PARA EL DESARROLLO SOSTENIBLE. Francia: UNESCO. https://es.unesco.org/themes/educacion-desarrollo-sostenible

Martinez Carrera S. Carnicero A. Carrera I. (2021), Educación para el desarrollo sostenible Pag. 816-827. 